\title{
Red de municipios contra el Síndrome Metabólico
}

Armando Aguirre Hervís ${ }^{a}$ - Rosa María Azamar Arizmendi ${ }^{b}$ - Arnulfo Camacho Alcantar_ Enrique A. Colar Gómez ${ }^{\mathrm{d}}$ - Marcelina García López ${ }^{\mathrm{e}}$ - Edit Rodríguez Romero ${ }^{\mathrm{f}}$ - Juan A Rodríguez Hernándezg - José Luis Castillo Hernández ${ }^{h}$

RESUMEN: El presente artículo presenta una propuesta de intervención desde los municipios, la prevención y control del Síndrome Metabólico (SM), cuya detección utilizan criterios nacionales e internacionales; para su abordaje se adoptan los ejes esenciales de la Atención Primaria de Salud. Asimismo, se presentan avances en materia de coordinación

\footnotetext{
a Licenciatura en Medicina. H. Ayuntamiento de Xalapa. Contacto: aguirre.hervis@gmail.com.

b Doctorado en Administración Pública. Instituto de Administración Pública A. C. Contacto: rosy 61@hotmail.com.

c Maestro adscrito a la Dirección de Salud Pública de SESVER. Contacto: arnca@hotmail.com.

d Instituto de Salud Pública, Universidad Veracruzana. Contacto: ecolar@uv.mx.

e Especialidad en Salud Pública. Adscrita a Servicios de Salud de Veracruz, perteneciente a la Dirección de Salud Pública. Línea de investigación: Salud Pública (Síndrome metabólico). Contacto: magarlo45@gmail.com.

${ }^{\mathrm{f}}$ Maestra en Salud Pública. Instituto de Salud Pública, Universidad Veracruzana. Contacto: edrodriguez@uv.mx.

g Maestro adscrito a la Dirección de Salud Pública de Servicios de Salud de Veracruz. Contacto: colfraimamibarrio@hotmail.com.

${ }^{\mathrm{h}}$ Doctorado en Ciencias de la Actividad Física y Deporte. Facultad de Nutrición, región Xalapa, Universidad Veracruzana. Contacto: jcastillo@uv.mx.
} 
intergubernamental, intersectorial e interinstitucional, entre los que destaca la constitución de la Red integrada, inicialmente, por Xalapa, Coatzacoalcos, Minatitlán, Jáltipan, Agua Dulce y Moloacán, municipios donde se estableció una Mesa Interinstitucional. A esta iniciativa, se han sumado instituciones gubernamentales, organizaciones no gubernamentales, representantes legislativos, sociedades de profesionistas y asociaciones civiles.

Palabras clave: Municipios; Síndrome Metabólico, red, Atención Primaria.

ABSTRACT: This article presents a proposal for intervention from the municipalities, the prevention and control of Metabolic Syndrome (SM), the detection of which uses national and international criteria; For its approach, the essential axes of Primary Health Care are adopted. Likewise, progress is made in intergovernmental, intersectoral and inter-institutional coordination, among which the constitution of the Network stands out, initially made up of Xalapa, Coatzacoalcos, Minatitlan, Jáltipan, Agua Dulce and Moloacán, municipalities where an Inter-institutional Table was established. This initiative has been joined by government institutions, non-governmental organizations, legislative representatives, professional societies and civil associations.

Keywords: Municipalities; Metabolic Syndrome; Network; Care; Primary Care.

\section{Introducción}

E

1 Síndrome Metabólico (SM) es un problema de salud pública mundial, caracterizado por: obesidad abdominal o central, glucosa alterada en ayunas, hipertensión arterial, elevación de triglicéridos y baja del colesterol de alta densidad (C-HDL) o colesterol bueno.(Barrera, et al, 2008)

Las personas con síndrome metabólico tienen cinco veces más el riesgo de presentar diabetes mellitus (DM); dos veces, el riesgo de enfrentar enfermedad cardiovascular; y de dos a cuatro veces, enfermedad vascular cerebral, y de tres a cuatro veces, infarto al corazón. La diabetes mellitus es la enfermedad más ligada al síndrome metabólico; la Encuesta Nacional de Salud y Nutrición (ENSANUT 2018) reportó que en México el porcentaje de la población de 20 años y más con diagnóstico previo de diabetes en ambos sexos fue de $10.3 \%$ que comparada con la ENSANUT 2012 de 9.2\%, lo cual representó un incremento de 1.1 puntos porcentuales, en números absolutos significó 2.2 millones de personas con diabetes, que sumados a los 6.4 


\section{Artículo - Ciencias de la Salud}

millones registrados en el 2012, hacen un total de 8.6 millones de personas mayores de 20 años y más con diabetes. En México, los 7 estados que registraron en 2018 las prevalencias más altas de diabetes por arriba de la media nacional de 10.3\% fueron: Campeche 14.0\%, Tamaulipas 12.8\%, Hidalgo 12.8\%, Ciudad de México 12.7\%, Nuevo León 12.6\%, Coahuila 12.3\% y Veracruz 11.9 \%. (González, López, Mesa, 2019; INSP,2012)

La conjunción de la obesidad, diabetes e hipertensión son la causa principal de que niños, niñas y jóvenes desarrollen Síndrome Metabólico en nuestro país, ocasionando 130 mil defunciones y representando un gasto en los servicios de salud de más de 10 mil millones de pesos al año. (Castelo, Arnold et al, 2011)

La educación para la salud y la acción anticipatoria son estrategias importantes para la prevención y disminución del síndrome metabólico en la población (Quadratin, 2012). Sin embargo, para lograr acciones anticipadas se requiere de un amplio conocimiento del contexto sociocultural y la identificación de los determinantes sociales de las comunidades. Estos datos se sistematizan mediante la aplicación de una Metodología de la Investigación Acción Participativa.

Ante esta situación, cobra importancia la estrategia de la Atención Primaria a la Salud (APS), no solo entendida como el primer eslabón de la cadena de atención dentro de un sistema de salud, sino como el cimiento principal para la prevención de enfermedades desde la participación familiar y comunitaria. En la Declaración de Astaná (Conferencia Mundial sobre Atención Primaria de Salud, 25 y 26 de octubre de 2018), en el inciso II se expresa:

Estamos convencidos de que el fortalecimiento de la atención primaria de la salud es el enfoque más inclusivo, eficaz y efectivo para la mejora de la salud física y mental de las personas, así como su bienestar social, y que la atención primaria de la salud es la piedra angular de un sistema de salud sostenible para la cobertura sanitaria universal (CSU) y los Objetivos de Desarrollo Sostenible (ODS) relacionados con la salud

y en el inciso III, continúan:

Seguiremos combatiendo la creciente carga de enfermedades no transmisibles, que provocan mala salud y muertes prematuras debido al consumo de tabaco, el consumo nocivo de alcohol, los modos de vida y comportamientos poco saludables, y la insuficiente actividad física y las dietas malsana[s]. (OMS/UNICEF, 2018) 
Lo anterior, sustenta la importancia de la participación de los municipios como la autoridad gubernamental por excelencia para el desarrollo e implementación de políticas de salud, que se vinculen con los niveles estatal y nacional, mediante programas que empoderen a la comunidad en educación para la salud y la prevención de un problema de salud tan grave como lo es el SM en la actualidad.

De acuerdo con los resultados de la ENSANUT 2018, la obesidad en México sigue en aumento, particularmente en el grupo de 12 a 19 años, en donde el 14.6\% de la población la presenta. Las entidades que registran los porcentajes más altos son: Colima con 24.9\%; Veracruz, 22.9\%; Quintana Roo, 22.8\%; Sonora, 22.2\%; Tabasco, 19.9\%; Campeche, 19.3\%; y Yucatán con 19.1\%. De estos 7 estados, 5 corresponden a la región sureste, liderados por Veracruz que ocupa el segundo lugar a nivel nacional. (INEGI, 2020)

El Instituto Mexicano para la Competitividad A.C. (2015), considera al sobrepeso y a la obesidad como un elemento que reduce la competitividad del país, dado que afecta la salud y calidad de vida de quienes la padecen, lo cual tiene un impacto en la dinámica familiar, impone costos elevados para su tratamiento y de su comorbilidad, y por otra reduce la productividad de los trabajadores representando retos considerables para las finanzas gubernamentales.

Por lo anterior, en el Estado de Veracruz en 2019 se generó una propuesta de combate al SM desde el nivel municipal, sustentada en la estrategia de Atención Primaria a la Salud (APS), motivando, por tanto, la conformación de la Red de Municipios contra el Síndrome Metabólico. En primera instancia fue conformada por los municipios de: Xalapa, Coatzacoalcos, Minatitlán, Jáltipan, Agua Dulce y Moloacán, y fue fortalecida con el apoyo y participación de la Secretaría de Educación de Veracruz (SEV), Universidad Veracruzana (UV) a través del Instituto de Salud Pública, la Secretaría de Desarrollo (SEDESOL), la LXV H. Legislatura del Congreso del Estado de Veracruz y la Sociedad Veracruzana de Salud Pública.

La voluntad política de los presidentes municipales se formalizó con la firma de la carta de intención entregada el 27 de julio de 2019 al Ejecutivo Federal y a la Comisión de Salud del Congreso Local y del Congreso de la Unión, asimismo, se informó al Subsecretario de Prevención y Promoción de la Salud Federal. Estos hechos cuentan con la validación y respaldo académico de la Asociación Mexicana para el Estudio Multidisciplinario del Síndrome Metabólico A. C. y de la Organización Panamericana de la Salud (OPS). 
Artículo - Ciencias de la Salud

\section{Objetivo general}

Lograr la participación de los ayuntamientos en el combate del SM en su territorio desde la Atención Primaria a la Salud y el abordaje de los determinantes sociales.

\section{Objetivos específicos}

- Promover entre los municipios participantes que los ayuntamientos realicen la detección estandarizada del SM a través de la capacitación en la medición de la circunferencia de cintura.

- Propiciar entre los municipios participantes el cambio de paradigma en la participación ciudadana por participación comunitaria para la prevención del SM, a partir de estrategias culturalmente pertinentes.

- Favorecer entre las personas detectadas con riesgo de SM el consumo de la dieta de la milpa evitando las bebidas azucaradas y productos ultraprocesados (PUP).

- Incrementar entre las personas detectadas con riesgo de SM su actividad física a través de estrategias que conlleven al ejercicio intervalado.

\section{Estrategia}

Se trata de una propuesta de intervención basada en elementos científicos, técnicos, jurídicos, administrativos y logísticos necesarios que permitan a los municipios participantes de la Red la implementación de una estrategia y una política pública para la disminución de la incidencia de Enfermedades Crónicas No transmisibles (ECNT), a través de la prevención y control del Síndrome Metabólico (SM).

Para la evaluación de los adultos se adopta la definición mundial consensuada del síndrome metabólico propuesta por la Federación Internacional de Diabetes (2006) por estar contenida en el Apéndice Normativo A de la "Norma Oficial Mexicana, NOM-015-SSA2-2010, para la prevención, tratamiento y control de la diabetes mellitus" (SSA, 2010). En niños, niñas y 
adolescentes se adoptan los criterios establecidos en Guía ALAD "Diagnóstico, control, prevención y tratamiento del Síndrome Metabólico en Pediatría”. (ALAD, 2009)

Se adopta la medida de la circunferencia de cintura por los individuos y las comunidades, como elemento de alerta del SM, proceso en el que las personas y el personal de salud realizan una detección inicial de posibles sujetos metabólicamente comprometidos que no respondan a las medidas alimentarias y de actividad física recomendadas; el instrumento indicado para su medición es una cinta métrica de fibra de vidrio en óptimas condiciones para su uso.

Para la estrategia de abordaje del SM, desde los municipios, se siguen los ejes esenciales de la Atención Primaria de Salud retomados por la OMS y el gobierno de México en la presentación del Informe Salud Universal en el Siglo XXI: 40 años de Alma Ata (OMS/OPS, 2019):

- El empoderamiento comunitario y su participación en el cuidado de su salud;

- la atención intersectorial de los determinantes sociales de la salud, y su promoción, y;

- la prevención y el diagnóstico temprano del síndrome Metabólico y sus comorbilidades, prioritariamente de las ECNT.

La coordinación intergubernamental, intersectorial e interinstitucional en los municipios, bajo la dirección de sus ayuntamientos y la participación central de las autoridades auxiliares municipales en las localidades, permitirá a los individuos, familias y comunidades acceder a información y recursos gubernamentales y no gubernamentales para la toma de medidas eficaces. La educación para la prevención y detección del SM es fundamental, una de las medidas fundamentales es la medición de la circunferencia de cintura, dado que la grasa abdominal se considera como un factor de riesgo para desarrollar SM; por lo cual cobra vital importancia el escenario que representan las instituciones de educación en todos los niveles, así como los programas de salud escolar y, justamente, es en educación básica donde, a través de la Ficha Individual Acumulativa (FIA), se genera información muy valiosa como es el registro de peso y talla a nivel nacional y un cuestionario sobre ECNT. (Secretaría de Educación de Veracruz, 2018)

Sin embargo, la FIA no considera el registro de la circunferencia de cintura, lo cual representaría un instrumento muy importante tanto para identificar a los escolares en riesgo de desarrollar síndrome metabólico, como para la sensibilización y educación para la prevención de este padecimiento, su replicabilidad a las familias, para posteriormente extenderlo a las comunidades circundantes (manzanas, barrios, colonias y localidades). Todo el proceso irá acompañado de una extensa campaña de comunicación comunitaria y social - 


\section{Artículo - Ciencias de la Salud}

con enfoque intercultural- que promocione la automedición periódica de la circunferencia de cintura, cuya actividad será reportada voluntariamente en las instituciones de salud del primer nivel de atención.

\section{Avances}

A continuación, se puntualizan los principales avances en materia de coordinación intergubernamental, intersectorial e interinstitucional encaminada hacia el fortalecimiento de la Política Pública de prevención y control del SM, así como lo realizado por los municipios participantes en el proyecto:

- Se crea la Red de Municipios contra el Síndrome Metabólico, formada inicialmente por Xalapa, Coatzacoalcos, Minatitlán, Jáltipan, Agua Dulce y Moloacán, que juntas suman más de un millón doscientos habitantes.

- Se establece una Mesa Interinstitucional contra el SM en Municipios Veracruzanos que elabora en conjunto un informe sobre el problema, el cual es entregado al presidente de la República. En dicho documento se evidencia la magnitud de la problemática, así como la voluntad para colaborar desde los municipios en su atención con un enfoque prioritariamente anticipatorio.

- La Mesa Interinstitucional para la Atención del Síndrome metabólico en Municipios Veracruzanos, elabora una propuesta de Convenio Marco para la Atención del Síndrome Metabólico entre dependencias estatales y los municipios de la Red.

- Se firma un convenio específico de colaboración entre el Ayuntamiento de Xalapa y la Secretaría de Educación del Estado de Veracruz para la Atención al Síndrome Metabólico.

- Se obtienen declaraciones de intención de diversas instituciones a fin de sumar esfuerzos contra este síndrome.

•El Dr. Cristian Morales, representante de OPS/OMS en México, destaca:

...esta iniciativa va en la dirección correcta porque parte en el ámbito correcto, desde el ámbito adecuado, desde el ámbito territorial, desde el ámbito local, allí donde justamente las autoridades 
se encuentran con la comunidad, porque tenemos que involucrar a todos, todos tenemos que ser parte de la solución, todos tenemos algo que hacer... (2019)

-El Ayuntamiento de Xalapa realiza el acto de lanzamiento formal del Programa de la Red de Municipios contra el Síndrome Metabólico.

- La Red de Municipios contra el Síndrome Metabólico cuenta con la asesoría y el acompañamiento de las siguientes instituciones: Universidad Veracruzana, las Presidencias de las Comisiones de Salud y de la Comisión Corredor Interoceánico, Zonas Libres y Desarrollo Económico del Congreso del Estado de Veracruz, Presidencia de la Comisión de Salud de la Cámara Federal de Diputados, Sociedad Veracruzana de Salud Pública, la Asociación Mexicana para el Estudio Interdisciplinario del Síndrome Metabólico (AMESI), la Presidencia de la Red de Universidades Mexicanas Promotoras de la Salud (RMUPS), las Secretarías de Salud Federal y la Secretaría de Educación del Estado de Veracruz, el Colectivo de Salud ELAMMéxico y Empresarios Unidos por el Bien Común.

- La Red Mexicana contra el Síndrome Metabólico recibió invitación de las autoridades de salud federal para participar en las reuniones del Grupo Intersectorial de Salud, Alimentación,

Medio Ambiente y Competitividad (GISAMAC) en el que participan: SEMARNAT, Secretaría de Salud, Subsecretaría de Autosuficiencia Alimentaria de la SADER, UNICEF, CIAT, CIMMYT, COFEPRIS, CONABIO, El Poder del Consumidor, FAO, IMSS, Instituto Nacional de Ciencias Médicas y Nutrición Salvador Zubirán, Instituto Nacional de Salud Pública, Organización Panamericana de la Salud, Dirección de Opciones Productivas de la Secretaría de Bienestar, SEGALMEX; Secretaría Ejecutiva del Sistema Nacional de Protección de Niñas, Niños y Adolescentes; Sistema Nacional DIF y UAM-Xochimilco y la Autoridad Federal del Corredor Interoceánico de Istmo de Tehuantepec.

- La Red recibió invitación formal del Centro Nacional de Programas Preventivos y Control de Enfermedades (CENAPRECE) a fin de participar en la elaboración de la "Estrategia Nacional para una Alimentación Justa, Saludable y Sustentable contra el Sobrepeso y Obesidad".

\section{Acciones municipales}

Debemos mencionar que cada presidente municipal y su equipo de colaboradores que participan en la iniciativa de la Red de Municipios contra el Síndrome Metabólico deciden cómo abordarlo en el territorio, de acuerdo con la estrategia basada en APS y solicita a los 


\section{Artículo - Ciencias de la Salud}

gobiernos estatal y federal su apoyo, sumándose estos a las acciones desarrolladas en los municipios. A continuación, se mencionan los avances de cada municipio participante:

Municipio de Xalapa:

Participa la Secretaría de Desarrollo Social del ayuntamiento, sus 3 programas prioritarios (el Combate al Síndrome Metabólico, Acción contra las Adicciones y Festibarrio). El programa específico contra el SM cuenta con acciones en todas sus subdirecciones y presupuesto para el año 2020. Se lanzó la primera fase del Programa de la Red de Municipios contra el Síndrome Metabólico el 5 de octubre de 2019, con presencia de la representación de OPS/OMS en México.

Municipio de Agua Dulce:

Inicia el programa en la comunidad del Palacio Municipal (se integran equipos en el ayuntamiento).

Municipio de Coatzacoalcos:

Se organiza la participación de más de 3,000 jefes de manzana. En coordinación con la Jurisdicción Sanitaria y Escuelas modelo en programas de salud escolar.

Municipio de Minatitlán:

El DIF y la Dirección de Salud Municipal dirigen el proceso con la colaboración de la Facultad de Medicina, campus Minatitlán, Universidad Veracruzana.

Municipio de Moloacán:

La Dirección de Salud Municipal impulsa un programa de Salud basado en APS y Salud Pública con el apoyo de la Facultad de Medicina de Minatitlán. 
Municipio de Jáltipan:

Entregó el informe al presidente de la República sobre el quehacer de la Red de Municipios contra el síndrome Metabólico.

\section{Conclusiones}

Dada su magnitud, trascendencia y vulnerabilidad se identifica al SM como un problema de Salud Pública planteando un abordaje multidisciplinario y transectorial, así como la implementación de una Política Pública para su prevención y control, planteando como escenario el municipio bajo la dirección de sus ayuntamientos y la participación central de las autoridades auxiliares municipales en las localidades.

Dada la importancia y el derecho que la ciudadanía posee en los procesos de planificación y abordaje de la atención, se adopta la Atención Primaria a la Salud, facilitando la integración de los recursos materiales, económicos, filosóficos, sociales y culturales, institucionales y comunitarios en la Promoción de la Salud. Se destacan los avances en la conformación de la Red tanto operativos como administrativos y legales.

\section{Referencias}

ALAD, Diagnóstico, control, prevención y tratamiento del Síndrome Metabólico en Pediatría,. Recuperado de: https://bit.ly/3eIdhJo.

Barrera, M. P., Pinilla, A. E., Cortés, E., Mora, G., Rodríguez, M. N. (2008). Síndrome metabólico: una mirada interdisciplinaria. Rev Colomb Cardiol., 15(3):111-26.

Castelo-Elías-Calles, L. Arnold-Domínguez, Y., Trimiño-Fleitas, A.A., De Armas-Rodríguez Y. (2011). Epidemiología y prevención del síndrome metabólico. Revista Cubana de Higiene y Epidemiología. Recuperado de: https://bit.ly/2XV8CgT.

El Síndrome Metabólico representa un gasto en Salud de 10 mil millones de pesos. 9 de agosto 2012. El texto original de este artículo fue publicado por la Agencia Quadratín. Disponible en: https://bit.ly/34YY1Ty

Global Conference on Primary Health Care: From Alma-Ata towards universal health coverage and the Sustainable Development Goals (218) declaration of Astana. Recuperado de: https://bit.ly/3axIbAy. 


\section{Artículo - Ciencias de la Salud}

González-Chávez, A., López-Espinosa, R., Mesa-Pérez, J.A. (2019) Manual Práctico para la detección y el tratamiento integral del Síndrome Metabólico. Alfil, 93-97.

IDF Consensus Worldwide Definition of the Metabolic Syndrome. Recuperado de: https://bit.ly/3byqgLm.

IMCO, (2015). Kilos de más. Pesos de menos: Los costos de la obesidad en México. Recuperado de: https://bit.ly/2XY3Lfa.

INEGI. Instituto Nacional de Salud Pública. Secretaría de Salud. Encuesta Nacional de Salud y Nutrición. Presentación de Resultados. (2018). Recuperado de: https://bit.ly/2VuHion.

Instituto Nacional de Salud Pública. Secretaría de Salud. Encuesta Nacional de Salud y Nutrición. Resultados Nacionales. (2012). Recuperado de: https://bit.ly/2KojN9R.

OMS, "Salud Universal en el Siglo XXI: 40 años de Alma-Ata". Informe de la Comisión de Alto Nivel. Edición revisada. Recuperado de: https://bit.ly/2VtR1v3.

OMS/UNICEF. Declaración de Astaná: Global Conference on Primary Health Care (2018). Recuperado de: https://bit.ly/2VT564b.

Secretaría de Educación del Estado de Veracruz, Fincha Individual Acumulativa. Recuperado de: https://www.sev.gob.mx/educacion-basica/esvisa/ficha-individual-acumulativa/

Secretaría de Salud, Norma Oficial Mexicana NOM-015-SSA2-2010, Para la prevención, tratamiento y control de la diabetes mellitus. Recuperado de: https://bit.ly/2VTB8wM.

Transcripción del mensaje del Dr. Cristian Morales Furhimann, representante de OPS/OMS en México con motivo del lanzamiento del Programa de la Red de Municipios contra el Síndrome Metabólico, realizado el 5 de octubre de 2019: tomado de: https://www.youtube.com/watch?v=yOuh6V8Mst0 